\title{
Fringe optimization for structured illumination super-resolution microscope with digital micromirror device
}

\author{
Xibin Yang*,†, Qian Zhu*, Zhenglong Sun*, Gang Wen*, Xin Jin*, \\ Linbo Wang*, Jialin Liu*, Daxi Xiong*** and Hui $\mathrm{Li}^{*}, \S$ \\ *Jiangsu Key Lab of Medical Optics \\ Suzhou Institute of Biomedical Engineering and Technology \\ Chinese Academy of Sciences \\ No. 88 Keling Street, Suzhou 215163, P. R. China \\ $\dagger$ University of Chinese Academy of Sciences \\ Beijing 100049, P. R. China \\ *xiongdx@sibet.ac.cn \\ §hui.li@sibet.ac.cn
}

Received 2 February 2019

Accepted 6 April 2019

Published 16 May 2019

\begin{abstract}
Structured illumination microscopy (SIM) is a promising super-resolution technique for imaging subcellular structures and dynamics due to its compatibility with most commonly used fluorescent labeling methods. Structured illumination can be obtained by either laser interference or projection of fringe patterns. Here, we proposed a fringe projector composed of a compact multiwavelength LEDs module and a digital micromirror device (DMD) which can be directly attached to most commercial inverted fluorescent microscopes and update it into a SIM system. The effects of the period and duty cycle of fringe patterns on the modulation depth of the structured light field were studied. With the optimized fringe pattern, $1.6 \times$ resolution improvement could be obtained with high-end oil objectives. Multicolor imaging and dynamics of subcellular organelles in live cells were also demonstrated. Our method provides a low-cost solution for SIM setup to expand its wide range of applications to most research labs in the field of life science and medicine.
\end{abstract}

Keywords: Structured illumination; super-resolution; digital micromirror device; fringe pattern; modulation depth.

\section{Introduction}

Variations of Fluorescent microscopies are widely used as noncontact, minimally invasive imaging tools in molecular and cell biological studies. The resolution of traditional fluorescent microscopy is

$\S_{\text {Corresponding author. }}$

This is an Open Access article published by World Scientific Publishing Company. It is distributed under the terms of the Creative Commons Attribution 4.0 (CC-BY) License. Further distribution of this work is permitted, provided the original work is properly cited. 
limited by the Abbe diffraction limit which hinders its observation of subcellular structures at the nanoscale. In the last 20 years, several different kinds of super-resolution fluorescent microscopy techniques were developed, including Stimulated Emission Depletion microscopy (STED),${ }^{1-3}$ STochastic optical reconstruction microscopy (STORM), ${ }^{4,5}$ Photo-activated localization microscopy (PALM), ${ }^{6,7}$ and Structured illumination microscopy (SIM), , etc. Among them, SIM is most attractive in live cell dynamic research due to reduction of photobleaching and photodamage, as well as its compatibility with most fluorescent labeling dyes and fast imaging speed. ${ }^{10-13}$

In SIM, a striped light field at the focused sample plane is required to excite the fluorescent samples. The structured light could result in shifting of unobservable high spatial frequencies of the sample to the frequency-acceptable passband of the imaging system. The orientations and phases of the striped light field need to be switched, thereby yielding several raw images used for reconstruction of the final super-resolution image. In most commercial SIM systems, structured light fields were generated by the interference of two or three laser beams at the sample plane. In order to get the highest modulation depth of the striped patterns, the direction, focusing position and polarization of beams need to be critically controlled. To achieve orientation rotation and phase shifting of the structured light field, sophisticated methods using mechanical motion of grating or liquid crystal spatial light modulators were invented. ${ }^{14,15}$ These requirements make SIM instruments very complicated, requiring to be regularly maintained even for commercial products.

The structured illumination can also be generated by projecting periodic fringe patterns at the sample plane. Different patterns on an LCOS or DMD device can be projected at the sample plane for optical section imaging ${ }^{16,17}$ In 2013, Ming Lei et al. demonstrated an SIM Device (DMD). ${ }^{18}$ The DMD has an array of millions of aluminum reflectors of micromirrors integrated on a memory chip, ${ }^{19-21}$ and each mirror can be individually addressed and switched to two stable states $\left(+12^{\circ}\right.$ and $-12^{\circ}$ with respect to "on" and "off" state). The DMD provides precise spatial and fast temporal control ability for the generation of illumination patterns. Binary fringe patterns can be loaded onto the DMD chip and the chip is conjugated to the sample plane so that a periodic striped light field could be generated to excite the sample. However, this image projection is also diffraction limited. As the period of the fringe pattern decreases, so does the modulation depth of the striped light field at the image plane. The problem becomes even more serious with high numerical aperture objective. Hence, there is a demand for optimizing the fringe pattern for DMD-based SIM in order to get high quality reconstructed super-resolution images.

In this paper, we designed a DMD fringe projector which could be easily attached to an inverted fluorescent microscope and turn it into a structured illumination super-resolution microscope. Patterns with different duty cycle of the binary stripes were loaded on DMD to achieve the best modulation depth. Best quality of the final reconstructed images were obtained with the duty cycle of 1:12. Superresolution images of fluorescent beads and Bovine pulmonary arterial endothelial (BPAE) cells were demonstrated with $1.5 \times$ resolution improvement. Multi-color imaging was realized using a self-developed 9-wavelength high brightness LED array as excitation light source. Our system provides a lowcost solution to achieve fast super-resolution imaging and would be widely available to most research labs in the field of life science and medicine.

\section{Experimental Setup}

\subsection{DMD fringe projector}

The schematic diagram of our DMD-projectionbased SIM system is shown in Fig. 1. The light emitted from the high brightness LEDs with switchable wavelengths was coupled into an optical fiber (diameter $3 \mathrm{~mm}$, NA 0.4, U-LLG150, Olympus) and then irradiated on a programmable DMD chip (DLP7000, $1024 \times 768$ micromirrors, pixel pitch $13.68 \mu \mathrm{m}$, Texas Instruments, USA). Each micromirror of the DMD can be set on either $+12^{\circ}$ or $-12^{\circ}$ direction, corresponding to "on" or "off" status, to guide the reflected light either into or away from the optical path, respectively. The light modulated by the DMD was collimated by an achromatic collimating lens $(f=500.0 \mathrm{~mm}$, AC508500-A, Thorlabs Inc.) and guided into the back entrance of an inverted fluorescent microscope. The fringe pattern was projected onto the sample by a $100 \times$ objective (Apo, NA1.40, Olympus, Japan). The total demagnification factor of the projection 


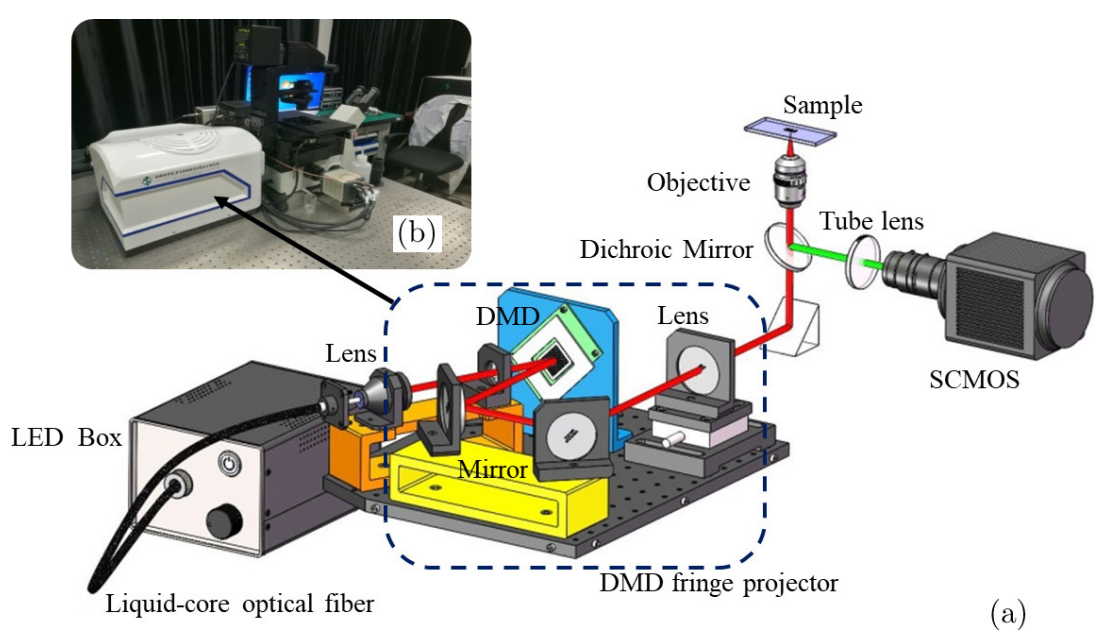

Fig. 1. Scheme (a) and photograph (b) of the DMD fringe projector attached to an inverted microscope.

system was $270 \times$. A high sensitivity sCMOS camera (ORCA Flash 4.0, Hamamatsu, Japan) was used to capture the fluorescent images. The DMD and sCMOS camera were synchronized via TTL trigger signal.

\subsection{Multi-wavelength LED excitation source}

A 9-wavelength high brightness LED array was developed as the excitation source. As shown in Fig. 2, nine vertical structured LED chips with different wavelengths $(370,385,405,450,472,525$, 570,635 , and $665 \mathrm{~nm}$ ) are packaged on one copper substrate $(23 \mathrm{~mm} \times 30 \mathrm{~mm})$ with efficient heat dissipation efficiency. The upper surface of each vertical structured LED chip is cathode. The back surface of all chips is packaged on a common anode with gold thread. Each chip can be controlled with the independent cathode and could be switched on or off independently, which enables the LED either to emit a single-band output or multi-band output simultaneously. A "Lumicite" Logo was labeled on the substrate. The size of each chip was $1 \times 1 \mathrm{~mm}^{2}$. The nine wavelengths cover the excitation spectrum of most commonly used fluorescent agents for molecular and cell biology. The output power of each chip was measured using an optical power meter (S130C, Thorlabs Inc., USA) as listed in Table 1.

\subsection{SIM imaging and data processing}

A set of binary fringe patterns with two perpendicular orientations and three phases for each orientation corresponding to $0,1 / 3 \pi$, and $2 / 3 \pi$ were generated with Matlab program and loaded onto DMD sequentially. So a total of six raw images were captured to reconstruct one super-resolution image. The reconstruction process was performed using the ImageJ plugin fairSIM developed by Müller et al. ${ }^{22}$

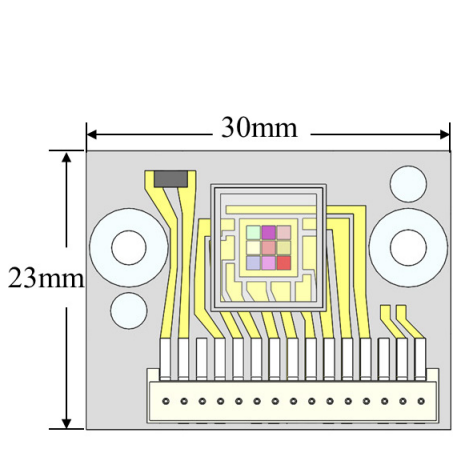

(a)

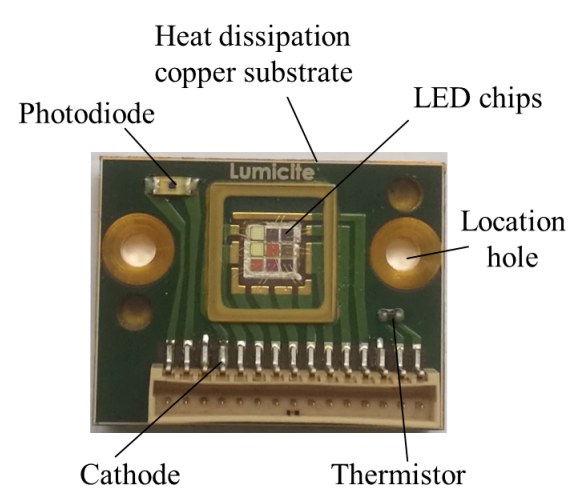

(b)

Fig. 2. (a) Schematic and (b) photograph of the self-developed multi-wavelength LED array excitation source. 


\section{Yang et al.}

Table 1. Wavelengths of the self-developed multi-wavelength LED array and the corresponding fluorescent agents.

\begin{tabular}{lcccl}
\hline Nominal wavelength $(\mathrm{nm})$ & Main wavelength $(\mathrm{nm})$ & FWHM $(\mathrm{nm})$ & Power $(\mathrm{mW})$ & Applicable fluorescent agents \\
\hline 370 & 368.9 & 16 & 1400 & Fura-2, Coumarins, Alexa 350, .. \\
385 & 387 & 14 & 1400 & DAPI, Hoescht, Fura-2, Qdots, .. \\
405 & 403.1 & 19 & 1500 & Alexa 405, wt GFP, Cascade Blue, ... \\
450 & 454.8 & 24 & 1580 & CFP, FuraRed $\left(\right.$ Ca $\left.{ }^{++}\right)$, Alexa $430, \ldots$ \\
472 & 471.5 & 27 & 1250 & Alexa 488, Cy2, Oregon Green, $\ldots$ \\
525 & 522.8 & 35 & 535 & YFP, Alexa 514, 532, Eosin, ... \\
570 & 589.8 & 22 & 450 & DsRed, TRITC, Cy3, Texas Red, .. \\
635 & 632.1 & 17 & 680 & Cy5, Alexa 635, 633, 647, TOTO-3, .. \\
665 & 660.5 & 20 & 635 & TruRed, Alexa 660, 680, Cy5.5, ... \\
\hline
\end{tabular}

As comparison, the wide field (WF) images were captured with all DMD micromirrors turned "on".

\section{Optimization of the Fringe Pattern on DMD}

Fringe patterns on the DMD were projected at the sample plane passing through a demagnifying imaging system including a collimating lens and a $100 \times$ oil objective. The higher orders of spatial frequencies of the binary fringe are naturally blocked by the imaging system thereby leading to a nearsinusoidal striped light field at the sample plane. The spatial frequency of the obtained pattern depends on the period of the fringe pattern on DMD. The white and dark pixels in Fig. 3(a) represent the "on" and "off" status of the micromirrors of DMD. The intensity distribution at sample plane can be calculated by convolution of the binary patterns and the illumination PSF of the system.
As shown in Fig. 3(b), the distribution curve demonstrates a near-sinusoidal distribution, which can be described by the equation:

$$
I(x)=I_{0}+I_{1} * \cos (2 \pi f x) .
$$

Here, $f$ is the spatial frequency of the stripped light field, $I_{0}$ is the average light intensity which is adjusted to the same value in the Fig. 3(b). The maximum intensity is $I_{0}+I_{1}$, and the minimum intensity is $I_{0}-I_{1}$. The modulation depth $D$ can be calculated as:

$$
D=2 * I_{1} /\left(I_{0}+I_{1}\right) .
$$

The dependence of modulation depth and the theoretical resolution on the fringe period is plotted in Fig. 3(c). It demonstrates that higher spatialfrequency patterns yield a higher-resolution reconstructed image. However, higher spatial-frequency patterns also cause low modulation depth of the striped light field at the sample plane. For a larger period of DMD patterns, the resolution (FWHM) of

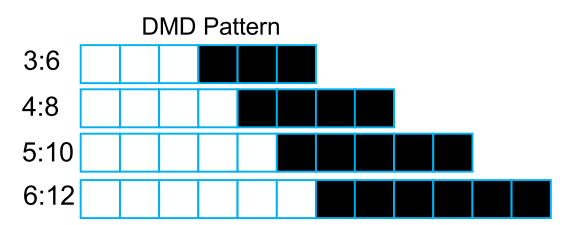

(a)

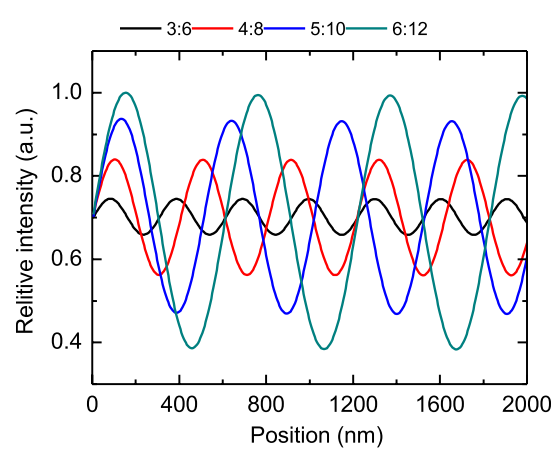

(b)

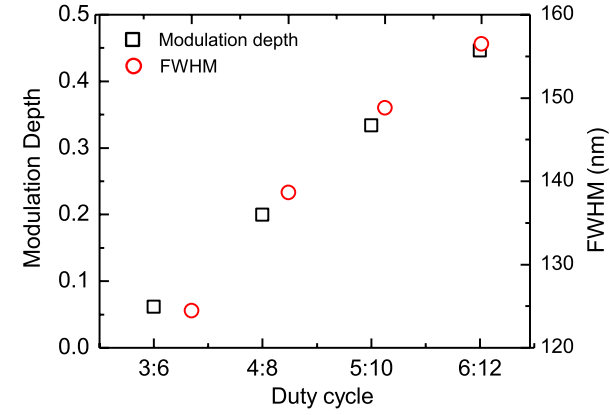

(c)

Fig. 3. Evaluation of the fringe period on the modulation depth and image resolution. (a) Binary patterns loaded on the DMD chip with different periods; (b) normalized intensity distribution obtained at the focal plane of the objective; (c) modulation depth and resolution (FWHM) with different fringe patterns. 
the system is worse, but the modulation depth increases with the increasing period of DMD patterns. A tradeoff has to be made to balance the resolution and modulation depth. Generally, raw images with modulation depth lower than 0.3 would induce low SNR and unavoidable artifacts of the reconstructed image. ${ }^{22}$ The modulation depth close to 0.5 could be obtained for the pattern with a duty cycle of 6:12 which is suitable for SIM superresolution reconstruction.

Figure 4 shows that the decrease of the number of the "on" pixels for one period, i.e., only one DMD-mirror is set to 'on' status in the DMD pattern, can further improve the modulation depth of the pattern compared with Fig. 3. Meanwhile, the modulation depth increases with the decreasing of duty cycle. The modulation depth is higher than 0.5 for the pattern with duty cycle of 1:10, 1:11 and $1: 12$. Note that the modulation depth lower than 0.5 will result in low SNR, and the probability of the artifacts is very high as shown in fairSIM (open source ImageJ plugin for SR-SIM reconstructions). In order to consider the quality of the final reconstructed images with better resolution and SNR, a period of 12 pixels with the duty cycle of $1: 12$ in DMD is used to turn an inverted fluorescent microscope into a structured illumination superresolution microscope. The theoretical modulation depth is 0.63 and the theoretical resolution of the reconstructed image is about $156.5 \mathrm{~nm}$ for the emission wavelength of $680 \mathrm{~nm}$.

The modulation depth can be further increased by changing the duty cycle even with the same period. Figure 5(a) shows the fringe patterns with a duty cycle from $6: 12$ to $1: 12$. Figure $5(\mathrm{~b})$ shows the corresponding intensity distribution at the sample plane. All patterns induced near-sinusoidal distribution with the same period of $608 \mathrm{~nm}$, but modulation depth varied from 0.45 to 0.63 . The smaller duty cycle, the higher the modulation depth. We also measured the intensity distribution with fluorescent beads at the same condition, shown in Fig. 5(d). The experimentally measured modulation depth in Fig. 5(e) has the same trend with the calculated modulation depth in Fig. 5(c), but the values are smaller which may result from additional background noise and imperfect optics of the imaging system.

It should be pointed out that the transmittance of the whole system is also directly related with the duty cycle, which effect the recorded fluorescence intensity and eventually the imaging speed. With duty cycle of $1: 12$, the transmittance was about $2.5 \%$ for our instrument, as measured by the ratio of light power out of the objective over the power shining on DMD device. With used high brightness LED light sources, reasonable fluorescence intensity can be still recorded with camera exposure time of $50 \mathrm{~ms}$.

\section{Experimental Results}

\subsection{Characterizing the resolution}

To demonstrate the super-resolution capabilities of the DMD-projection-based SIM system, fluorescent beads (diameter: $50 \mathrm{~nm}$, excitation wavelength: $640 \mathrm{~nm}$ and emission wavelength: $680 \mathrm{~nm}$ ) were imaged with a $640 \mathrm{~nm}$ exciting wavelength of LED source. Figure 6 shows the obtained WF and SIM super-resolution images. The beads in Fig. 6(b) not only looks much smaller than in Fig. 6(a) but also distinguishable in clusters. The line profile of two

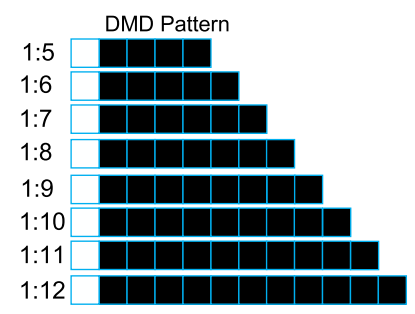

(a)

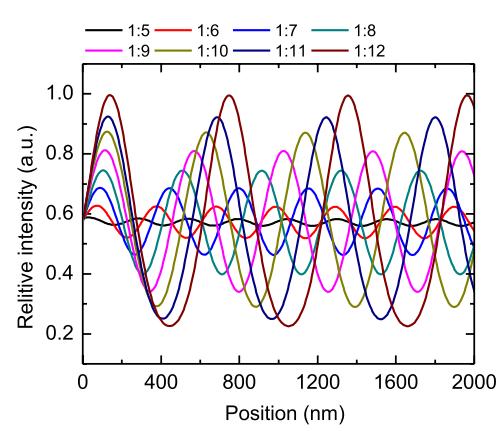

(b)

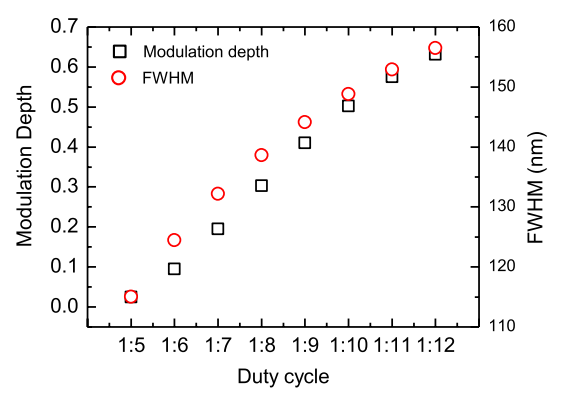

(c)

Fig. 4. Generation of sinusoidal fringe illumination with different duty cycles by numerical calculation. (a) Binary patterns loaded on the DMD chip with different duty cycles, (b) the normalized intensity distribution obtained in the focal plane of the objective and (c) the corresponding calculated modulation depth and resolution (FWHM) with different obtained fringe patterns. 


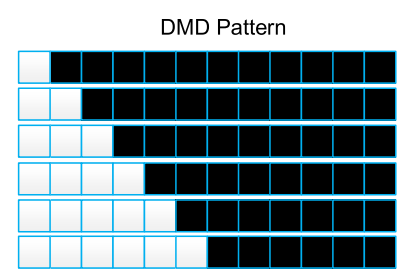

(a)

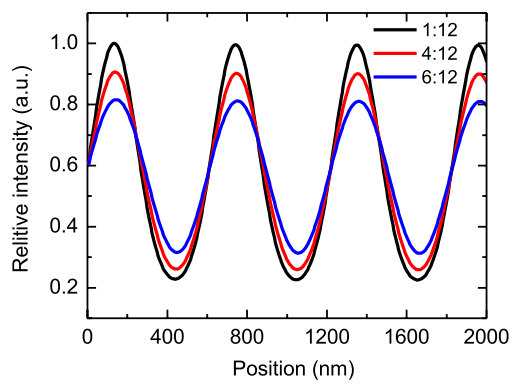

(b)

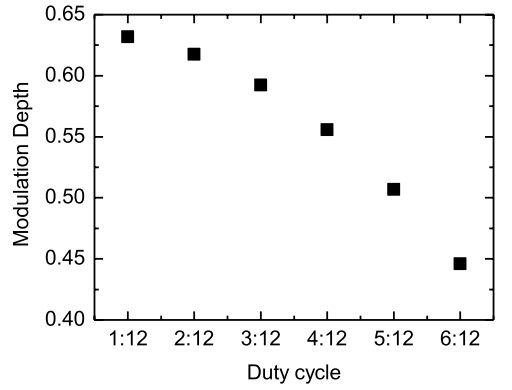

(c)

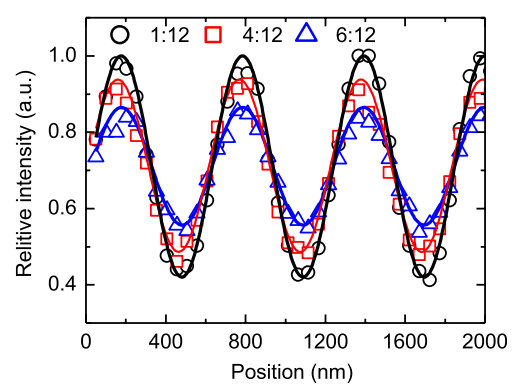

(d)

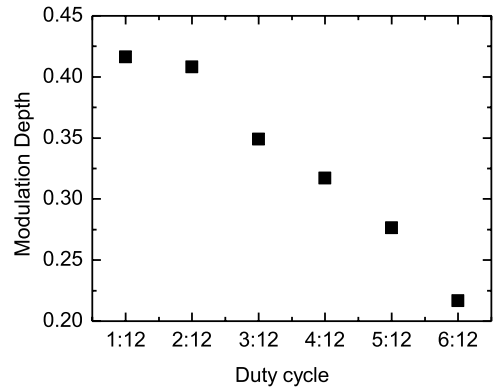

(e)

Fig. 5. Evaluation of the different duty cycle but same period on the modulation depth. (a) Binary patterns loaded on the DMD chip; (b), (c) the calculated normalized intensity distribution obtained at the focal plane of the objective and modulation depth with different duty cycle; (d), (e) the corresponding intensity distribution and modulation depth experimentally measured in our setup.

neighboring beads was just like a big blob in the WF but clearly separated into two peaks in SIM, as shown in Fig. 6(c). The line profiles of a single bead are close to Gaussian distribution for both WF and SIM image but have different FWHM, shown in Fig. 6(d). The measured FWHM from 30 beads in
SIM is $190 \pm 6 \mathrm{~nm}$, while the FWHM in WF is $312 \pm 6 \mathrm{~nm}$. Thus $1.6 \times$ spatial resolution improvement was obtained with our setup, benefitted from the optimized fringe pattern.

Just as shown in Fig. 5, the modulation depth can be further increased by changing the duty cycle
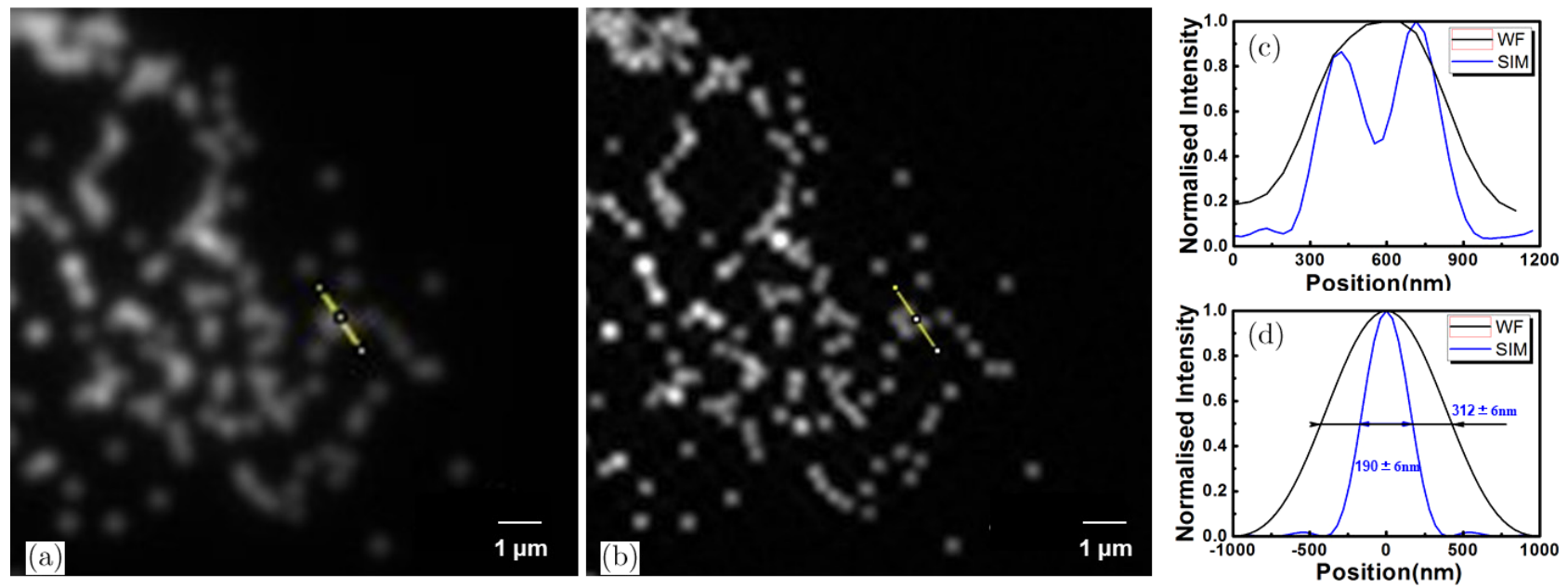

Fig. 6. Characterizing the resolution of the DMD-projection-based SIM system with $50 \mathrm{~nm}$ fluorescent beads. (a) Conventional wide-field (WF) image; (b) reconstructed SIM super-resolution image; (c) line profile of two neighboring beads which are not distinguishable in WF but clearly separated in SIM; (d) line profile of a single beads shows the FWHM in SIM is $1.6 \times$ times smaller than that in WF. 


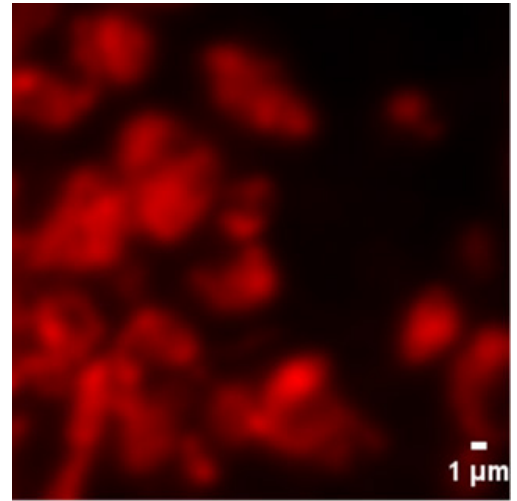

(a)

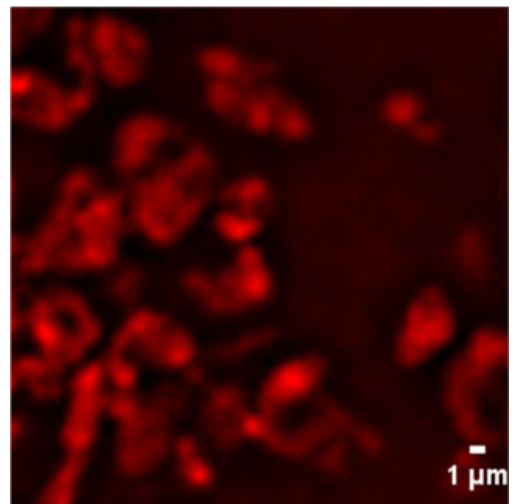

(b)

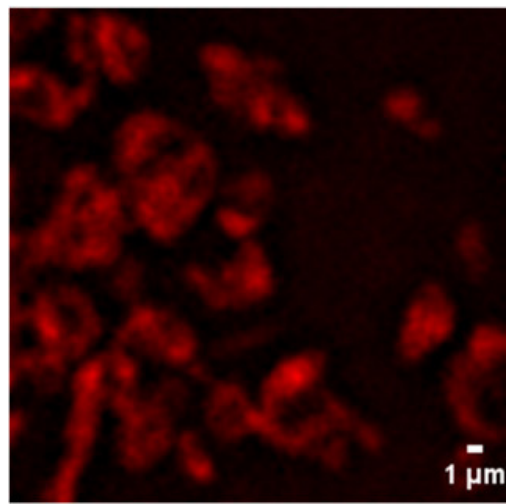

(c)

Fig. 7. Comparison of the reconstructed images under different duty cycles. (a) wide field image; (b) image reconstructed under duty cycle of $6: 12$; (c) image reconstructed under duty cycle of 1:12.

even with the same period. To demonstrate the influence of different duty cycles on the image SNR, a comparison of the reconstructed images under different duty cycles are shown in Fig. 7. The images were captured using the mitochondrion in BPAE cells under the excitation wavelength of $560 \mathrm{~nm}$. The mitochondrion structure is much clearer in SIM image, each one can be easily distinguished. While in the wide field image, the microstructures were smeared out. This resolution improvement could be helpful to study the cell skeleton structural change in the process of cell secretion, migration, division, and fusion. The images captured with duty cycle of 6:6 and 1:12 are shown. Due to higher modulation depth, more detailed feature of mitochondrion structure could be distinguished in Fig. 7(c) captured with duty cycle of 1:12 than Fig. 7(b) captured with duty cycle 6:6.

\subsection{Multicolor imaging}

Multicolor fluorescent imaging is essential to study the interactions of different organelles in a cell. Our SIM setup employed a nine-wavelength LED array as excitation light source which enables multi-color imaging by simply turning on/off the individual LED chip. We demonstrate three-color superresolution imaging with trichrome-stained BPAE cells (Thermo Fisher Scientific Inc.). Mitochondria of the cells were labeled with MitoTracker Red CMXRos, F-actin was labeled with Alexa Fluor 488 phalloidin, and nuclei was labeled with DAPI. The samples were sequentially illuminated by the wavelengths of $405 \mathrm{~nm}, 472 \mathrm{~nm}, 570 \mathrm{~nm}$ to obtain multi-color images. Figure 8 shows the superimposed images of the trichrome-stained BPAE cells in WF (a) and SIM (b).
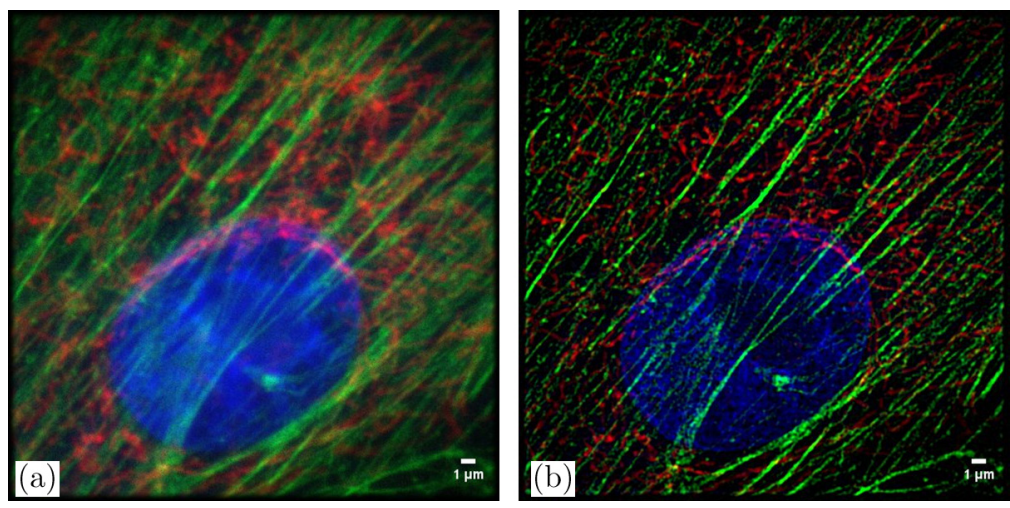

Fig. 8. Multi-color super-imposed WF (a) and SIM (b) images of trichrome-stained BPAE cells. Mitochondria are indicated in red, F-actins in green and nuclei in blue. 

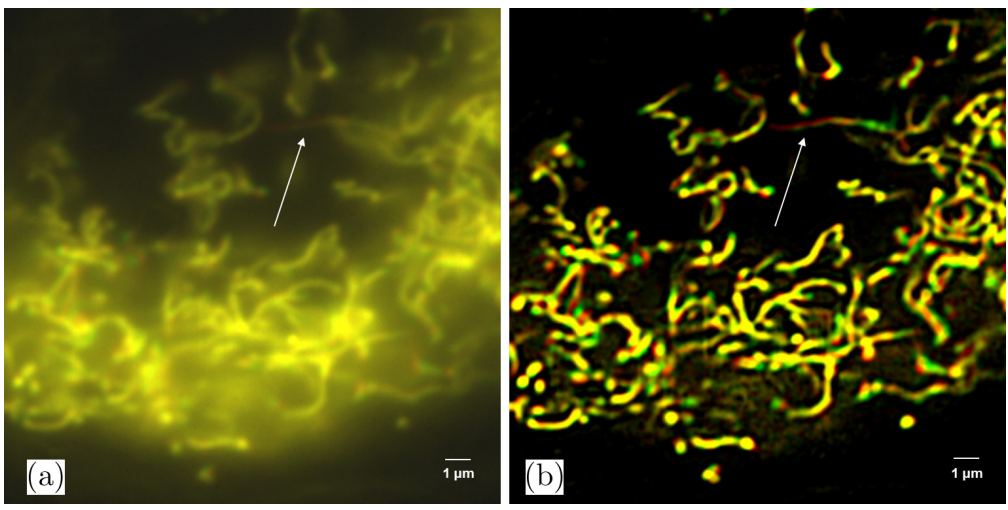

Fig. 9. Dynamic WF(a) and SIM(b) imaging of mitochondria in live Cos7. The images were rendered with different colors from green to red correspond to time series. The colorful feature in (b) clearly demonstrates the movement of the mitochondria.

\subsection{Dynamic imaging with live cells}

The imaging speed of SIM depends on time response to change the illumination pattern and the camera exposure time. Due to the fast switching capability of the DMD device more than $100 \mathrm{kHz}$, the time response to change the fringe pattern can be neglected in our system. Hence the imaging speed is only limited by the exposure time. Considering the typical fluorescence exposure time of $10 \mathrm{~ms}$ per image, the imaging speed of this system could be up to $16 \mathrm{fps}$. Figure 9 (Supplementary Videos 1 and 2) shows the dynamic imaging of mitochondria images in live Cos7 cells. To clearly observe the dynamic process of mitochondria, an overlay of the images were rendered from red to green according to the time series, as shown in Figs. 9(a) and 9(b). The colorful feature in Fig. 9(b) pointed by the arrow shows the movement of the mitochondria. Fusion of a few mitochondria can also be clearly seen in the SIM video but not in WF video. The reconstructed image clearly demonstrates the ability of our SIM system to record fine movements of organelles at the subcellular level.

\section{Conclusion}

We developed a DMD fringe projector which can be attached to a commercial inverted fluorescent microscope and enable it for SIM super-resolution imaging. Fringe patterns were optimized to balance the resolution and modulation depth. $1.6 \times$ resolution improvement was demonstrated with an oil immersion objective. The system could be applied to multi-color imaging and dynamic imaging with a frame rate up to 16 fps. Our system is low cost, easy to be installed and maintained. So it could be available to the most biological lab for daily observation of sub-cellular structures and cell dynamics.

\section{Acknowledgments}

The study was funded by the National Key Technologies R\&D Program of China (2018YFC0114800 and 2017YFC0109900), the Natural Science Foundation of China (NSFC) (61405238), the Natural Science Foundation of Jiangsu Province (BK20141206), and the Key Technologies R\&D Program of Jiangsu Province (BE2018666).

\section{References}

1. S. W. Hell, "Far-field optical nanoscopy," Science 316(5858), 1153-1158 (2007).

2. S. W. Hell, J. Wichmann, "Breaking the diffraction resolution limit by simulated emission: Stimulatedemission-depletion fluorescence microscopy," Opt. Lett. 19(11), 780-782 (1994).

3. X. Yang et al., "Sub-diffraction imaging of nitrogenvacancy centers in diamond by stimulated emission depletion and structured illumination," RSC Adv. 4 (22), 11305-11310 (2014).

4. M. J. Rust et al., "Sub-diffraction-limit imaging by stochastic optical reconstruction microscopy (STORM)," Nat. Meth. 3(10), 793-796 (2006).

5. B. Huang et al., "Three-dimensional superresolution imaging by stochastic optical reconstruction microscopy," Science 319(5864), 810-813 (2008).

6. E. Betzig et al., "Imaging intracellular fluorescent proteins at nanometer resolution," Science 313(5793), 1642-1645 (2006). 
7. S. T. Hess et al., "Ultra-high resolution imaging by fluorescence photoactivation localization microscopy," Biophys. J. 91(11), 42584272 (2006).

8. M. G. Gustafsson, "Surpassing the lateral resolution limit by a factor of two using structured illumination microscopy," J. Microscopy 198(2), 82-87 (2000).

9. A. G. York et al., "Resolution doubling in live, multicellular organisms via multifocal structured illumination microscopy," Nat. Meth. 9(7), 749-754 (2012).

10. L. Schermelleh et al., "Subdiffraction multicolor imaging of the nuclear periphery with 3D structured illumination microscopy," Science 320(5881), 13321336 (2008).

11. J. Fitzgibbon et al., "Super-resolution imaging of plasmodesmata using three-dimensional structured illumination microscopy," Plant Physiol. 153, 14531463 (2010).

12. P. J. Keller et al., "Fast, high-contrast imaging of animal development with scanned light sheet-based structured-illumination microscopy," Nat. Meth. 7, 637-642 (2010).

13. L. M. Hirvonen et al., "Structured illumination microscopy of a living cell," Eur. Biophys. J. 38(6), 807-812 (2009).

14. N. Bozinovic et al., "Fluorescence endomicroscopy with structured illumination," Opt. Exp. 16(11), 8016-8025 (2008).
15. B. J. Chang et al., "Isotropic image in structured illumination microscopy patterned with a spatial light modulator," Opt. Exp. 17(17), 14710-14721 (2009).

16. P. Křížek et al., "Flexible structured illumination microscope with a programmable illumination array," Opt. Exp. 20(22), 24585-24599 (2012).

17. D. Xu et al., "Fast optical sectioning obtained by structured illumination microscopy using a digital mirror device," J. Biomed. Opt. 18(6), 060503 (2013).

18. D. Dan et al., "DMD-based LED-illumination superresolution and optical sectioning microscopy," Sci. Rep. 3, 1116 (2013).

19. J. Qian et al., "Full-color structured illumination optical sectioning microscopy," Sci. Rep. 5, 14513 (2015).

20. X. Zhou et al., "Image recombination transform algorithm for superresolution structured illumination microscopy," J. Biomed. Opt. 21(9), 096009 (2016).

21. V. Bansal, P. Saggau, "Digital micromirror devices: Principles and applications in imaging," Cold Spring Harb. Protocols 2013(5), 404-411 (2013).

22. M. Müller et al., "Open-source image reconstruction of super-resolution structured illumination microscopy data in ImageJ," Nat. Commun. 7, 10980 (2016). 